\title{
Influence of Tablet Splitting on Dissolution of Tablets with Naproxen Sodium
}

\author{
Dorota Wójcik-Pastuszka ${ }^{1}$, Kordian Juszkiewicz ${ }^{1}$, Gizem Özhan², and Witold Musiał ${ }^{1 *}$ \\ ${ }^{1}$ Department of Physical Chemistry, Wroclaw Medical University, Wroclaw, Poland \\ ${ }^{2}$ Department of Pharmaceutical Technology, Ankara University, Ankara, Turkey
}

\section{ABSTRACT}

e-mail: witold.musial@umed.wroc.pl

The aim of this paper was to examine the impact of mechanical tablet splitting on in vitro dissolution of naproxen sodium. Naproxen ( 250 and $500 \mathrm{mg}$ ) was used in the experiment. The in vitro tests were conducted using the USP paddle apparatus. The release of the drug from the 500-mg (whole 500-mg tablets or two 250-mg tablets), $250 \mathrm{mg}$ (whole 250-mg tablets or fragments obtained by halving a 500-mg tablet), and $125 \mathrm{mg}$ (fragments obtained by halving a 250-mg tablet or quartering a 500-mg tablet) formulations of naproxen in phosphate buffer solution $(\mathrm{pH}=6.8)$ were monitored spectrophotometrically at $271 \mathrm{~nm}$. The difference factor $\left(f_{1}\right)$ and the similarity factor $\left(f_{2}\right)$ for the obtained profiles were derived. The dissolution profiles were also analyzed using first-order, second-order, and Korsmeyer-Peppas model equations. The release rate constants, half release times, and correlation coefficients for these models were also calculated. Statistical methods including Student's t-test were employed for comparison of the dissolution profiles. Kinetic studies and statistical analysis suggested that splitting naproxen tablets did not alter the dissolution profile or pharmacokinetics, although the values obtained for $f_{1}$ and $f_{2}$ may indicate differences between the release from intact tablets and fragments containing the same amount of the drug.

KEYWORDS: naproxen, tablet splitting, dissolution profiles, difference factor, similarity factor, first-order kinetics, second-order kinetics, Korsmeyer-Peppas equation, Student's t-test

\section{INTRODUCTION}

T he splitting of oral dosage formulations has become a very popular and promising method in pharmacotherapy. Tablets are often divided into halves or even quarters to supply the proper dose of a drug for the patient, especially if low-dose preparations are not available. This possibility facilitates a treatment for patients, especially the elderly and children, who have difficulty swallowing and/or taking a whole tablet due to pain and discomfort. Moreover, the technique of tablet splitting allows for cost reductions (1). However, the process of dividing and crushing oral dosage forms might result in supplying an incorrect dose of the drug or weight loss (2). Depending on the active substance, it could promote harmful effects on the patient. The size of the tablet, its shape, and hardness might also play an important role in tablet division. Formulations containing drugs with a high therapeutic index and long half-life are recommended for tablet splitting operations (3). Scored tablets are regarded as safe for splitting; however, not all scored tablets can be precisely divided, which can cause dosage fluctuations. It was revealed that the method used for tablet splitting, such as hand breaking or using a tablet splitter or kitchen knife, impacts the exactness and stability of the obtained dosage form (1). van RietNales et al. reported that only hand broken tablets fulfilled requirements (4). Cook et al. observed that $16 \%$ of fragments obtained using a tablet splitter and $58 \%$ obtained with a kitchen knife were out of standard (5). These observations were confirmed by studying the impact of splitting techniques on lisinopril via physical data. It was observed that 37 out of 40 tablet fragments obtained by hand breaking varied above $10 \%$ from the mean weight, although only 3 out of 40 parts obtained from the splitter were different from the average weight. The discrepancy in the mass of the drug in the fragments affected the dissolution and drug content (6). Another author connected this inconsistency in the weight of tablets after splitting the surface, resulting from a fracture (7). In this work, $25 \%$ of the samples obtained manually were out of specification, in the terms of USP standard for the variability of the weight of tablets below $130 \mathrm{mg}$, which is established in the general monograph as $10 \%$. A microscopic investigation revealed that cracking

*Corresponding author. 
arose from a rugged area. This was more pronounced in the case of manual splitting compared to using a tablet splitter.

The study of the influence of tablet splitting on the content uniformity of tablets was performed by Vranić et al. (8). In this case, it was noticed that the manual method, as well as using a tablet splitter, did not influence the content uniformity of the tablets and complied with requirements. An in vitro release study of metoprolol succinate from split and whole tablets did show significant discrepancies between the corresponding dissolution profiles. Inconsistencies were only observed in the samples with lower dose strengths. This is probably due to limited breaking reproducibility with respect to the surface area and morphology. The investigation indicated that tablet splitting did not influence the kinetic model of the released substance $(9,10)$. Ishitsuka et al. examined the impact of tablet splitting on drug dissolution behaviors with theophylline sustained-release tablets (11). This showed a change in the drug release mechanism after breaking a tablet into two fragments, as the dividing process increased the drug release rate. This was explained by microscopic observation of the tablet fracture surface, which became sharp and irregular and displayed numerous cavities.

Tablet splitting can affect stability during storage. It was found that cardiovascular medications should not be split in advance. Drugs, such as digoxin, significantly degraded chemically within 30 days, which is very important in clinical practice (12); however, intact and split tablets containing gabapentin did not show any differences between the potency and dissolution when stored under normal conditions for nine weeks (13).

Considering all these data, it is clear that not all tablets can be divided. The process of splitting tablets depends on the formulation. In many cases, split tablets may cause weight deviations. The process could be useful when the required dose is not commercially available. An example of this would be with naproxen tablets, which are available in the Polish market at doses of 200, 250, and $500 \mathrm{mg}$. The dose for children older than 2 years of age is $10 \mathrm{mg} / \mathrm{kg}$ of body weight per day in two divided doses. This means that for children whose body weight is $20 \mathrm{~kg}$, a dose of $100 \mathrm{mg}$ naproxen is needed, which results in splitting tablets with higher doses.

The aim of this work was to test the impact of splitting naproxen tablets on the in vitro dissolution of the drug. We studied whether breaking tablets alters naproxen release pharmacokinetic parameters including the release rate constant, the half release time, and the dissolution mechanism.

\section{MATERIAL AND METHODS \\ Materials}

Hydrochloric acid $(\mathrm{HCl})$ was obtained from $\mathrm{POCH}$ SA (Gliwice, Poland); sodium phosphate tribasic dodecahydrate $(\mathrm{NaPO} \bullet 12 \mathrm{H} 2 \mathrm{O})$ and sodium hydroxide $(\mathrm{NaOH})$ were purchased from Chempur (Piekary Śląskie, Poland); 250- and 500-mg naproxen tablets were purchased from Hasco-Lek (Wrocław, Poland); and naproxen sodium was supplied as a gift from HascoLek (Wrocław, Poland). All synthetic chemicals were of analytical reagent grade, and all components were used as received.

\section{Instrumentation and Equipment}

The $\mathrm{pH}$ of the phosphate buffer solution was established using a CPC-511 pH/conductivity meter (ELMETRON, Zabrze, Poland). The spectrum for naproxen sodium and the absorbance of the released drug was monitored with a V-530 spectrophotometer (JASCO, Tokyo, Japan). The weights of the intact and divided tablets were obtained using a WAS 160/C/2 analytical balance (RADWAG, Poland). The USP II paddle apparatus DT-700 (ERWEKA, Germany) was applied in the in vitro dissolution study.

\section{Dissolution Test Conditions}

In vitro release studies were performed according to the European Pharmacopoeia (14) at $37 \pm 0.5{ }^{\circ} \mathrm{C}$ and a rotation speed of $50 \mathrm{rpm}$. Naproxen was released into $1000 \mathrm{~mL}$ of phosphate buffer solution at $\mathrm{pH}$ 6.8. Each dissolution test was conducted in six replicates (series $A$, $B, C, D, E$, and F) while maintaining the sink conditions. Samples $(3 \mathrm{~mL})$ were withdrawn at fixed time intervals and the dissolution medium was replenished with $3 \mathrm{~mL}$ of phosphate buffer. The dissolution study was conducted for 8 hours, so the release of naproxen sodium from the tablets was assessed in the expanded period compared to the usual timeframe. The release rate and fitting to the kinetic models was determined for a wide set of obtained data. We observed that the intact tablets and fragments of tablets were still releasing the drug after bypassing the recommended time of $45 \mathrm{~min}$. It was interesting both for the scientific reasons, as well as for the calculations, to recognize the course of the release in the entire time, until $100 \%$ of the drug was released

Naproxen was released from the tablets or tablet fragments reflecting 500, 250, or $125 \mathrm{mg}$ of the drug. The 500-mg dose strength was obtained from an intact 
500-mg naproxen tablet or two intact 250-mg tablets. The 250-mg dose strength was derived from one intact 250-mg naproxen tablet or a 500-mg tablet split into two fragments. Dividing 250-mg naproxen tablets in half or 500-mg naproxen tablets in quarters yielded the formulations for the 125-mg dose strength. The tablets were manually divided, and all the fragments were weighed.

\section{Analytical Procedures}

Phosphate buffer solution ( $\mathrm{pH}$ 6.8) was prepared according to the European Pharmacopoeia (14). Naproxen sodium was diluted in phosphate buffer solution to a concentration of $0.03125 \mathrm{mg} / \mathrm{mL}$ and the spectrum was measured at $298 \mathrm{~K}$. Four characteristic absorption maxima were observed at 262, 271, 312, and $330 \mathrm{~nm}$. The calibration curve was prepared at $271 \mathrm{~nm}$ using five different concentrations of naproxen sodium ranging from 0.03125 to $0.003906 \mathrm{mg} / \mathrm{mL}$ and reading the absorbance.

The absorbance of the released drug was measured at $271 \mathrm{~nm}$, and the concentration was calculated based on the prepared calibration curve. When the absorbance of the sample was above 1, it was diluted with phosphate buffer.

\section{Dissolution Data Evaluation}

The naproxen release values from the intact tablets and fragments were fitted to the following equations.

First-order kinetics model:

$$
\ln \left(m_{0}-m_{t}\right)=\ln m_{0}-k_{1} t
$$

where $m_{0}$ is the amount of drug in the formulation before the dissolution, $m_{t}$ is the amount of the drug released over time $t$, and $k_{1}$ is the first-order release rate constant.

Second-order kinetics model:

$$
\frac{1}{\mathrm{~m}_{0}-\mathrm{m}_{\mathrm{t}}}=\frac{1}{\mathrm{~m}_{0}}-\mathrm{k}_{2} \mathrm{t}
$$

where $k_{2}$ is the second order rate constant.

Korsmeyer-Peppas model $(15,16)$ :

$$
\log \frac{\mathrm{m}_{\mathrm{t}}}{\mathrm{m}_{\infty}}=\log \mathrm{k}_{\mathrm{K} \cdot \mathrm{p}}+\mathrm{n} \log \mathrm{t}
$$

where $m_{\infty}$ is the amount of drug released after an infinite amount of time (in this study after 8 hours), $k_{\text {K-p }}$ is the Korsmeyer-Peppas rate constant, and $\mathrm{n}$ is the parameter indicative of the drug release mechanism.

Based on these equations, the release rate constants, half release time, and the " $n$ " parameter were derived.

To compare the release profiles, the difference factor $\left(f_{1}\right)$ and the similarity factor $\left(f_{2}\right)$ were calculated with the following equations $(17,18)$ :

$$
\begin{gathered}
\mathrm{f}_{1}=\frac{\sum_{\mathrm{t}=1}^{\mathrm{n}}\left|\mathrm{R}_{\mathrm{t}}-\mathrm{T}_{\mathrm{t}}\right|}{\sum_{\mathrm{t}=1}^{\mathrm{n}} \mathrm{R}_{\mathrm{t}}} \times 100 \\
\mathrm{f}_{2}=50 \times \log \left\{\left[1+\frac{\sum_{\mathrm{t}=1}^{\mathrm{n}}\left(\mathrm{R}_{\mathrm{t}}-\mathrm{T}_{\mathrm{t}}\right)^{2}}{\mathrm{n}}\right]^{-0.5} \times 100\right\}
\end{gathered}
$$

where $\mathrm{n}$ is the number of time points, $\mathrm{R}_{\mathrm{t}}$ is the dissolution value of the reference batch at time $t$, and $T_{t}$ is the dissolution value of the test batch at time $t$.

An $f_{1}$ value ranging from $0-15$ and an $f_{2}$ value ranging from 50-100 signify sameness or equivalence of the two profiles.

\section{Statistical Analysis}

The linearity of the kinetic models was evaluated by linear regression analysis, which was calculated via the leastsquares regression method. The best fitting kinetic model was selected based on the comparison of the standard deviation (SD) and the correlation coefficient, $R^{2}$. The drug release profiles were assessed statistically using Student's t-test. A statistically significant difference was indicated when $\mathrm{p}<0.05(19,20)$.

\section{RESULTS AND DISCUSSION}

The amount of the drug released from the intact 250-mg naproxen tablet and from the formulation obtained by splitting the 500-mg naproxen tablet into two parts over 8 hours is presented in Figure $1 a$ and $1 b$, respectively. The variability of dissolution profiles shown in Figure 1a was rather low, according to the RSD values calculated for every time point; the RSD was in the range from 0.02 to -0.07 , with exception of the first time point, which was 0.1 . The obtained RSD range suggests that all the tablets $(A, B, C, D, E$, and $F)$ used in the test had the very similar amounts of the drug at the initial stage, and the amounts released over time were also close to each other. However, 
the dissolution profiles presented in Figure 1B were more varied; RSD values in the range of $0.14-0.17$ indicate that the formulations obtained by dividing the 500-mg naproxen tablet contained varying amounts of the drug. There was more variation in the amount of drug released from each part of the tablet at the same time, compared to the intact tablets. The same results were observed when dividing the 500-mg naproxen tablets into four fragments and dividing the $250-\mathrm{mg}$ naproxen tablets into two fragments. The calculated mass and percentage of naproxen in all the fragments used in this study are listed in Table 1. The formulations derived from the division of the 500-mg tablets in two parts contained 194.8 to 297.2 mg of naproxen, which corresponds to $77.9-118.9 \%$ of the required weight, respectively. Only two fragments were very close to $250 \mathrm{mg}$, specifically the 248.8 and $248.6 \mathrm{mg}$ fragments. Similar discrepancies were also observed in other cases. Among the 18 formulations obtained in the splitting process, nine fragments did not comply with the Pharmacopoeia requirements, meaning that they were not in the tablet drug content range of $85-115 \%$ (21, 22). These results are consistent with results obtained by Cook et al., where splitting tablets containing $10 \mathrm{mg}$ of drug yielded fragments comprising 2.46 to $7.48 \mathrm{mg}$ of the active substance (5).
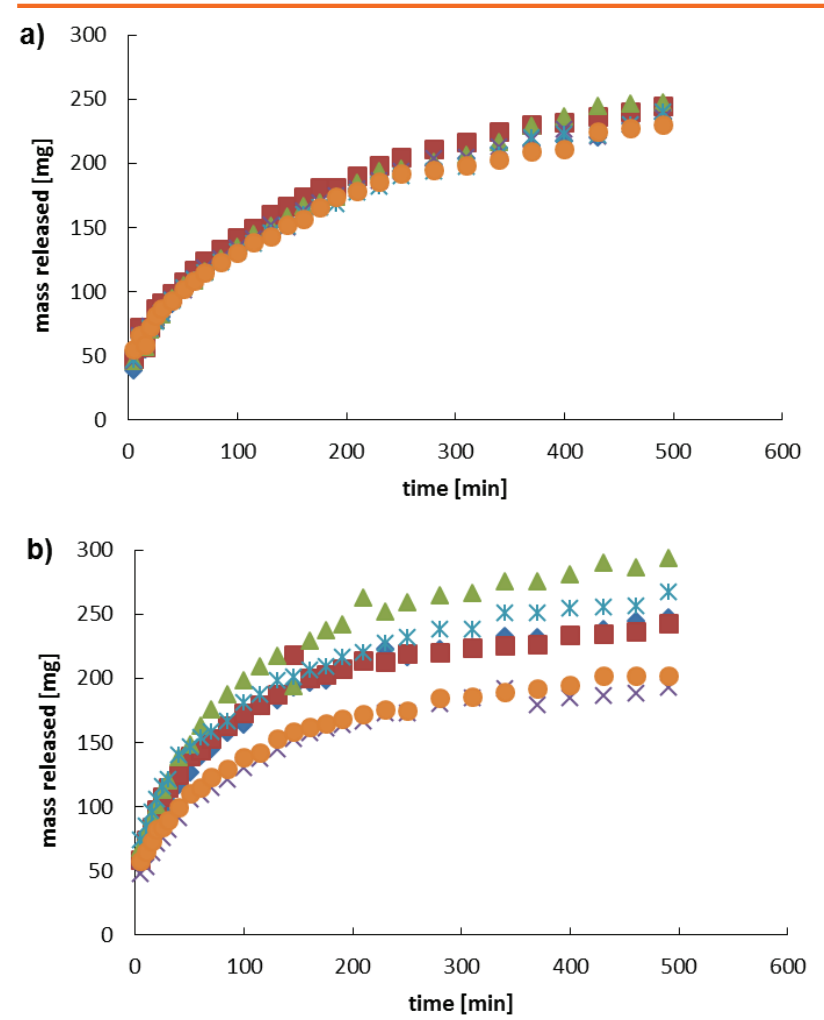

Figure 1. Dissolution profiles for (a) intact 250-mg naproxen tablets and (b) 500-mg naproxen tablet fragments $(n=6)$.

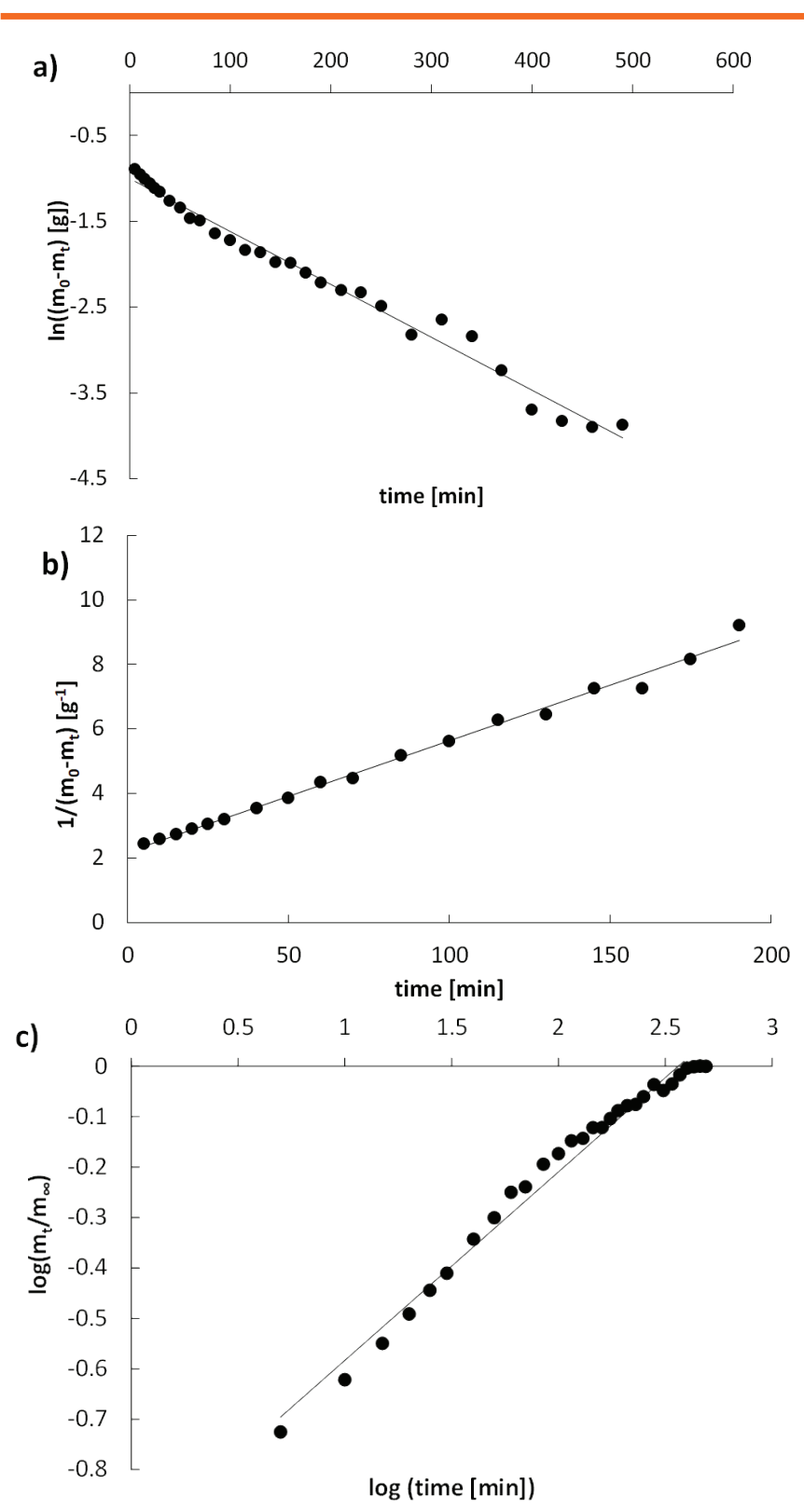

Figure 2. Drug release pharmacokinetics for the 500-mg intact naproxen tablet using the (a) first-order model, (b) second-order model, and (c) Korsmeyer-Peppas model.

The dissolution profiles were fit to first-order, secondorder, and Korsmeyer-Peppas kinetic models (Fig. 2). The release parameters, such as the first-order rate constant $k_{1}$, second-order rate constant $k_{2}$, Korsmeyer-Peppas rate constant $k_{k-p}, n$ coefficient, and half release time $t_{0.5}$, for all the models were derived and are listed in Table 2. The first-order plots were fairly linear, as indicated by the high correlation coefficient $R^{2}$ ranging from 0.9267 to 0.9818 (Fig. 2a). Second-order kinetics only worked for the time ranging from 0 to $190 \mathrm{~min}$ (Fig. 2b). In this time range, the parameter $R^{2}$ values were high, ranging from 0.9380 to 0.9933 . To study the mechanism of drug release from these formulations, the data were fit according to the 
Korsmeyer-Peppas equations, with exemplification on Fig. 2c. The regression values $R^{2}$ were close to 1 and ranged from 0.9790 to 0.9914 . This result implied that the Korsmeyer-Peppas equations best described the release of naproxen from the formulations. The tablet splitting process did not significantly affect the pharmacokinetics of naproxen dissolution; however, the slight differences may suggest an influence of tablet splitting on drug release, as presented on Figure 3.

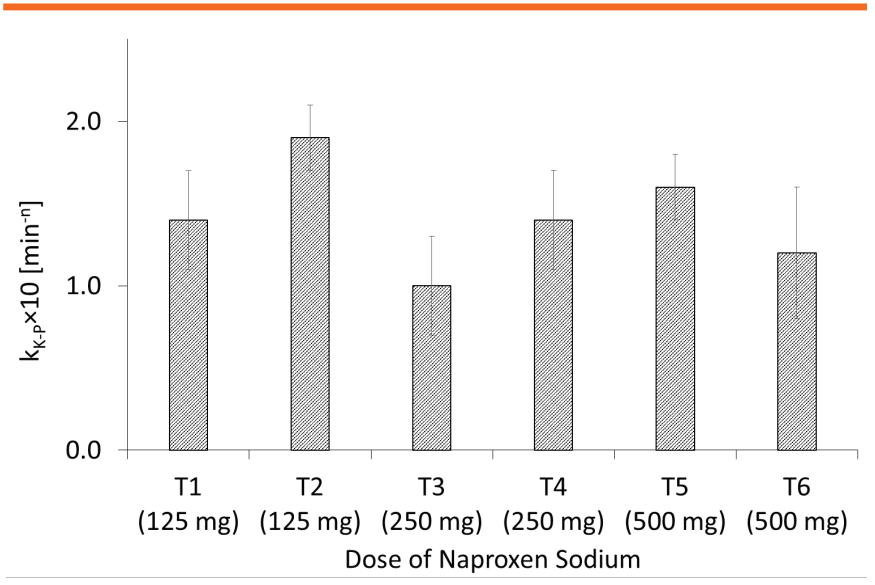

Figure 3. Comparison of release rate constants $\left(K_{K-p}\right)$ calculated with the Korsmeyer-Peppas equation for assessed combinations of intact and fragments of naproxen sodium tablets. T1, $125 \mathrm{mg}$ (1/4 of 500-mg tablet); T2, $125 \mathrm{mg}$ (1/2 of 250-mg tablet); T3, $250 \mathrm{mg}$ (1 250-mg tablet); T4, $250 \mathrm{mg}$ (1/2 of 500-mg tablet); T5, $500 \mathrm{mg}$ (2 250-mg tablets); T6, $500 \mathrm{mg}$ (1 500-mg tablet).

Moreover, in all cases, the values of the parameter $n$, which characterizes the transport mechanism, were similar, meaning that the mechanism of drug release from the intact and divided tablets was the same. The obtained mean $\pm S D$ value for the diffusional exponent, $\mathrm{n}$, ranged from $0.23 \pm 0.01$ to $0.37 \pm 0.02$, suggesting that the release was governed by Fickian diffusion; in all assessed cases the value of $\mathrm{n}$ in Korsmeyer-Peppas equation is below 0.5 (16). The pharmacokinetic parameters $\mathrm{k}_{\mathrm{K}-\mathrm{p}}$ and $\mathrm{t}_{0.5}$, calculated using the KorsmeyerPeppas equation for the same dose strength, were within the standard deviation. The same results were obtained in the study examining the influence of divided tablets on metoprolol succinate dissolution in which tablet splitting did not impact the release mechanism or the amount of drug released (9). However, in the present research, the values for the first-order kinetic model rate constant and half release time indicated slight discrepancies for the different formulations at the same dose (Table 2). These discrepancies can arise from the kinetics equation, which did not sufficiently describe the studied process. Another explanation may be the influence of the surface on the pharmacokinetic parameters. It has been reported that the drug release rate was higher after dividing theophylline tablets (11). That research demonstrated that the tablet surfaces were uneven and irregular with empty spaces after splitting, which extended the length of the dissolution test; however, it may also be because the naproxen tablets disintegrated immediately after introduction into the acceptor fluid. Although tablet disintegration is observed, the drug is gradually dissolved in the medium.

The dissolution profiles obtained for the same doses with various formulations are presented in Figure 4. The comparisons were performed by calculating $f_{1}$ and $f_{2}$. The results are shown in Table 3. Generally, $f_{1}$ values below 15 and $f_{2}$ values greater than 50 indicate similarity or equivalence between the two curves $(18,20)$. Based on the results from Table 3, there were no differences between the compared profiles obtained for the 125

(Fig. 4a) and 500-mg doses (Fig. 4c). In the case of the 250-mg dose, the $f_{1}$ value was slightly higher than 15 (19.75) and the $f_{2}$ value was slightly lower than 50 (46.23). According to Fig. 4b, the drug was released slightly faster from the fragments obtained by splitting the 500-mg tablet into two parts compared with the intact 250-mg tablets. This suggests that the initial rough and uneven surface area of the dosing unit obtained after tablet splitting was better exposed to the dissolution medium. Figure 4a shows similar data because both entities are split and have a rough and uneven surface area.

In contrast, in the work of Takka et al. (23), the derived $f_{2}$ values were 42.2 and $\mathbf{4 7 . 7}$ for the split and intact tablets, respectively. Takka concluded that although these $f_{2}$ values ( 42.2 and 47.7 ) were below 50 , there were no differences between the dissolution profiles (23).

The similarity of the profiles presented in Fig. 4a may demonstrate that both 125 -mg doses, either obtained by splitting a 250-mg tablet into two parts or a 500-mg tablet into four parts, interacted with the acceptor fluid in the same way. The lack of differences between the curves in Fig. $4 \mathrm{~b}$ means that the dissolution medium had the same influence on drug release from the $500 \mathrm{mg}$ dose with two intact 250-mg tablets and the single 500-mg tablet.

Another method allowing for the comparison of the dissolution profiles is a statistical method, such as Student's t-test (20). The calculated t-values were 0.05 , 0.31 , and 0.13 for the 125,250 and $500 \mathrm{mg}$ doses, respectively. The tabulated value was 2.04 at the $95 \%$ confidence level. The obtained t-values were lower than the tabulated t-value, meaning that there were no differences between the compared profiles. 
Table 1. Weight of Drug and its Percentage in Fragments Obtained by Dividing Naproxen Tablets

\begin{tabular}{|c|c|c|c|c|c|c|c|c|c|}
\hline \multicolumn{2}{|c|}{ Dose Strength } & A & B & C & D & E & $\mathbf{F}$ & Mean & RSD (\%) \\
\hline \multirow{4}{*}{$250 \mathrm{mg}^{\mathrm{a}}$} & Intact Tablet (mg) & \multicolumn{2}{|c|}{552.0} & \multicolumn{2}{|c|}{548.2} & \multicolumn{2}{|c|}{550.2} & 550.1 & 0.34 \\
\hline & Fragment (mg) & 274.7 & 274.5 & 325.9 & 213.6 & 310.5 & 234.5 & 267.8 & 15.02 \\
\hline & Drug (mg) & 248.8 & 248.6 & 297.2 & 194.8 & 282.2 & 213.1 & 247.5 & 14.41 \\
\hline & Drug (\%) & 99.5 & 99.4 & 118.9 & 77.9 & 112.9 & 85.2 & 99.0 & 14.42 \\
\hline \multirow{4}{*}{$125 \mathrm{mg}^{\mathrm{b}}$} & Intact Tablet (mg) & \multicolumn{2}{|c|}{272.6} & \multicolumn{2}{|c|}{277.0} & \multicolumn{2}{|c|}{274.3} & 274.6 & 0.8 \\
\hline & Fragment (mg) & 118.1 & 151.8 & 115.9 & 158.9 & 149.7 & 121.7 & 136.0 & 13.1 \\
\hline & Drug (mg) & 108.3 & 148.3 & 104.6 & 143.4 & 136.4 & 110.9 & 125.3 & 14.2 \\
\hline & Drug (\%) & 86.6 & 118.6 & 83.7 & 114.7 & 109.1 & 88.7 & 100.2 & 14.2 \\
\hline \multirow{4}{*}{$125 \mathrm{mg}^{\mathrm{c}}$} & Intact Tablet (mg) & \multicolumn{4}{|c|}{547.7} & \multicolumn{2}{|c|}{546.8} & 547.2 & 0.1 \\
\hline & Fragment (mg) & 155.0 & 110.7 & 128.6 & 152.2 & 149.3 & 124.0 & 136.6 & 13.2 \\
\hline & Drug (mg) & 141.5 & 101.1 & 117.4 & 138.9 & 136.5 & 113.4 & 124.8 & 13.2 \\
\hline & Drug (\%) & 113.2 & 80.88 & 93.9 & 111.1 & 109.2 & 90.7 & 99.8 & 13.2 \\
\hline
\end{tabular}

a, obtained by halving a 500-mg tablet; $b$, obtained by halving a 250-mg tablet; c, obtained by dividing a 500-mg tablet into four parts. RSD, relative standard deviation.

Table 2. Pharmacokinetic Models and Parameters for Naproxen Release from Intact Tablets and Fragmented Tablets

\begin{tabular}{|c|c|c|c|c|c|c|c|}
\hline \multirow{3}{*}{ Models } & \multirow{3}{*}{ Parameters } & \multicolumn{6}{|c|}{ Evaluated Dose } \\
\hline & & \multicolumn{2}{|c|}{$125 \mathrm{mg}$} & \multicolumn{2}{|c|}{$250 \mathrm{mg}$} & \multicolumn{2}{|c|}{$500 \mathrm{mg}$} \\
\hline & & $\begin{array}{l}\text { Fragment of } \\
\text { 250-mg Tablet }\end{array}$ & $\begin{array}{l}\text { Fragment of } \\
\text { 500-mg Tablet }\end{array}$ & $\begin{array}{c}\text { One Intact } \\
\text { 250-mg Tablet }\end{array}$ & $\begin{array}{l}\text { Fragment of } \\
\text { 500-mg Tablet }\end{array}$ & $\begin{array}{l}\text { Intact 500-mg } \\
\text { Tablet }\end{array}$ & $\begin{array}{c}\text { Two intact } \\
\text { 250-mg Tablets }\end{array}$ \\
\hline \multirow{3}{*}{ F-O } & $\begin{array}{l}\mathrm{k}_{1} \times 10^{3} \\
\mathrm{~min}^{-1}\end{array}$ & $6.1 \pm 0.2$ & $7.9 \pm 0.4$ & $5.6 \pm 0.3$ & $7.0 \pm 0.8$ & $5.7 \pm 0.3$ & $4.0 \pm 0.3$ \\
\hline & $R^{2}$ & 0.9640 & 0.9818 & 0.9733 & 0.9267 & 0.9769 & 0.9699 \\
\hline & $\begin{array}{c}\mathrm{t}_{0.5} \\
\mathrm{~min}\end{array}$ & $116.5 \pm 4.7$ & $87.7 \pm 4.2$ & $128.8 \pm 6.8$ & $97.0 \pm 9.0$ & $121.9 \pm 7.0$ & $173.6 \pm 11.5$ \\
\hline \multirow{3}{*}{ S-O } & $\begin{array}{c}\mathrm{k}_{2} \times 10^{2} \\
\mathrm{~g}^{-1} \mathrm{~min}^{-1} \\
\end{array}$ & $8.8 \pm 0.3$ & $9.7 \pm 0.5$ & $4.1 \pm 0.2$ & $9.0 \pm 1.0$ & $3.1 \pm 2.1$ & $2.2 \pm 0.2$ \\
\hline & $R^{2}$ & 0.9933 & 0.9919 & 0.9911 & 0.9380 & 0.9824 & 0.9858 \\
\hline & $\begin{array}{l}\mathrm{t}_{0.5} \\
\mathrm{~min}\end{array}$ & $96.0 \pm 4.0$ & $72.0 \pm 3.0$ & $99.1 \pm 4.6$ & $46.5 \pm 4.8$ & $65.0 \pm 4.1$ & $93.0 \pm 5.2$ \\
\hline \multirow{4}{*}{ K-P } & $\begin{array}{l}\mathrm{k}_{\mathrm{K}-\mathrm{p} \times 10} \times 10 \\
\mathrm{~min}^{-\mathrm{n}}\end{array}$ & $1.9 \pm 0.2$ & $1.4 \pm 0.3$ & $1.0 \pm 0.3$ & $1.4 \pm 0.3$ & $1.2 \pm 0.4$ & $1.6 \pm 0.2$ \\
\hline & $\mathrm{n}$ & $0.23 \pm 0.01$ & $0.33 \pm 0.02$ & $0.37 \pm 0.02$ & $0.32 \pm 0.02$ & $0.35 \pm 0.02$ & $0.31 \pm 0.01$ \\
\hline & $R^{2}$ & 0.9913 & 0.9790 & 0.9894 & 0.9816 & 0.9835 & 0.9914 \\
\hline & $\begin{array}{l}\mathrm{t}_{0.5} \\
\mathrm{~min}\end{array}$ & $34.0 \pm 11.0$ & $46.0 \pm 20.0$ & $72.0 \pm 32.0$ & $44.0 \pm 16.0$ & $55.0 \pm 26.0$ & $47.0 \pm 15.0$ \\
\hline
\end{tabular}

F-O, first-order model; S-O, second-order model; K-P, Korsmeyer-Peppas model

Table 3. Difference Factor $\left(f_{1}\right)$ and Similarity Factor $\left(f_{2}\right)$ Values Calculated for Mean In

Vitro Dissolution Profiles $(n=6)$

\begin{tabular}{|c|c|c|}
\hline Dose strength $(\mathrm{mg})$ & $\boldsymbol{f}_{1}$ & $\boldsymbol{f}_{2}$ \\
\hline $\mathbf{1 2 5}$ & 5.36 & $\mathbf{7 1 . 5 4}$ \\
\hline $\mathbf{2 5 0}$ & 19.75 & 46.23 \\
\hline $\mathbf{5 0 0}$ & $\mathbf{7 . 6 8}$ & 65.19 \\
\hline
\end{tabular}



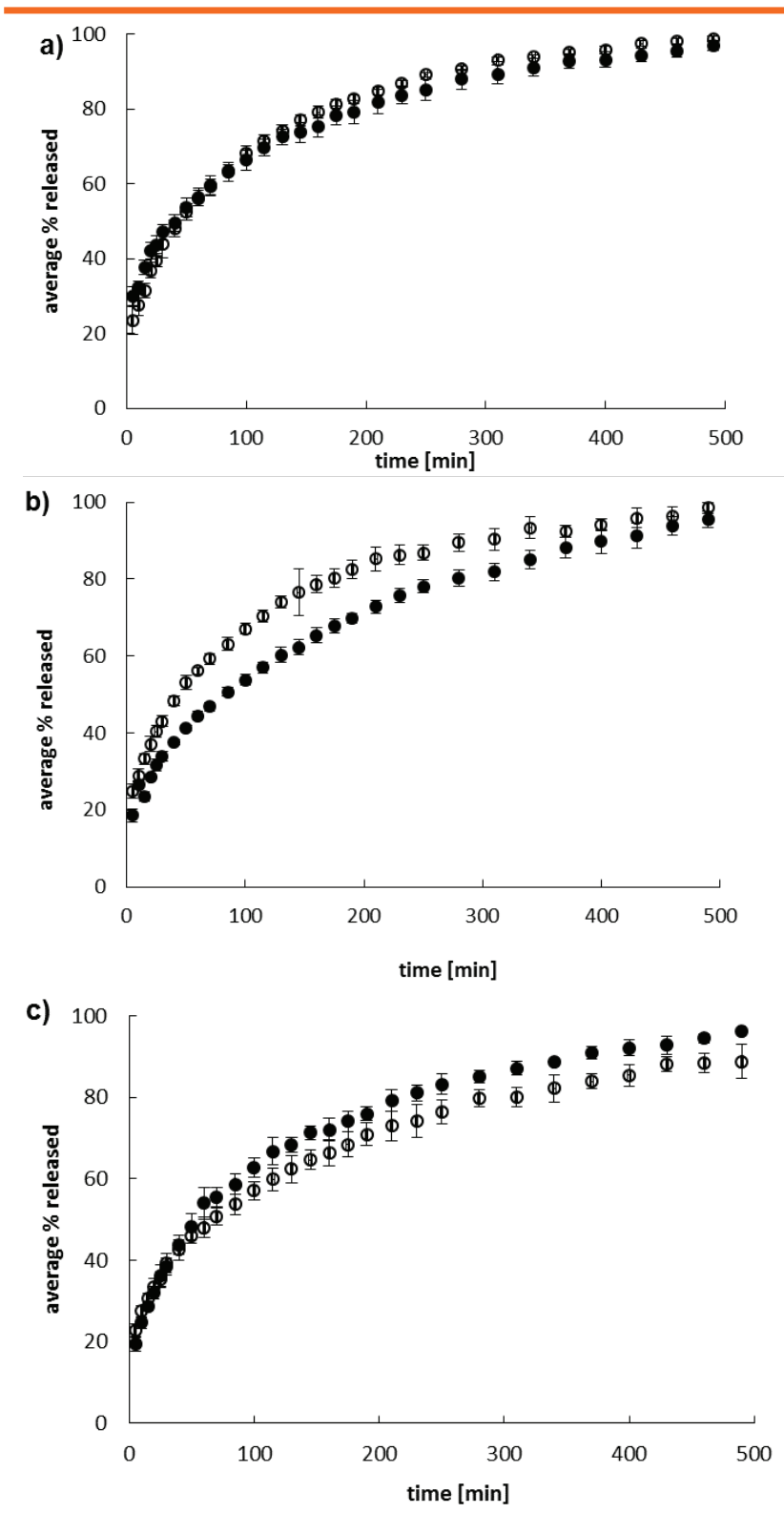

Figure 4. Mean dissolution profiles for the drug release from (a) 500-mg naproxen tablet split into four fragments (0) and 250-mg naproxen tablet split into two fragments (•); (b) intact 250-mg naproxen tablet $(\bullet)$ and 500-mg naproxen table split into two fragments (o); and (c) intact 500-mg naproxen tablet $(\bullet)$ and two intact $250-m g$ naproxen tablets (०).

\section{CONCLUSION}

In conclusion, the tablet splitting process yielded formulations consisting of various amounts of the drug, sometimes significantly different from the therapeutic dose. The release of naproxen from the intact and fragmented tablets was well described by KorsmeyerPeppas equations, while the transport of the drug from all formulations occurred according to Fickian diffusion. Tablet splitting did not change the mechanism of naproxen release. The release of naproxen from the tablet fragments was slightly faster than from the intact tablets containing the same amount of drug, as the dissolution medium could penetrate the sharp and irregular surface area easier.

\section{ACKNOWLEDGMENTS}

This work was supported by a grant from Wroclaw Medical University and the Ministry of Science and Higher Education, ST-950.

\section{CONFLICT OF INTEREST}

The authors declare that there are no conflicts of interest.

\section{REFERENCES}

1. Mosena, M. S.; Van der Merve, E. The appropriateness and risks of tablet splitting. S. A. Pharm. J. 2009, 76, 30-36.

2. van Santen, E.; Barends, D. M.; Frijlink, H. W. Breaking of scored tablets: a review. Eur. J. Pharm. Biopharm. 2002, 53, 139-45. DOI: 10.1016/S0939-6411(01)00228-4.

3. Helmy, S. A. Tablet splitting: is it worthwhile? analysis of drug content and weight uniformity for half tablets of 16 commonly used medications in the outpatient setting. J. Manag. Care Pharm. 2015, 21, 76-86. DOI: 10.18553/jmcp.2015.21.1.76.

4. van Riet-Nales, D. A; Doeve, M. E.; Nicia, A. E.; Teerenstra, S.; Notenboom, K.; Hekster, Y. A.; Van den Bemt, B. J. F. The accuracy, precision and sustainability of different techniques for tablet subdivision: breaking by hand and the use of tablet splitters or a kitchen knife. Int. J. Pharm. 2014, 466, 44-51. DOI: 10.1016/j. ijpharm.2014.02.031.

5. Cook, T. J.; Edwards, S.; Gyemah, C.; Shah, M.; Shah, I.; Fox, T. Variability in tablet fragment weights when splitting unscored cyclobenzaprine $10 \mathrm{mg}$ tablets. J. Am. Pharm. Assoc. 2004, 44, 583-586. DOI: 10.1331/1544-3191.44.5.583.

6. Fahelelboom, M. S. K; Al-Tabakha, M. M. M.; Eissa, N. A. M.; Javadi, J. Evaluation of certain pharmaceutical quality attributes of lisinopril split tablets. Sci. Pharm. 2016, 84, 646-653. DOI: 10.3390/scipharm84040646.

7. Habib, W. A.; Alanizi, A. S.; Alanizi, F. K. Accuracy of tablet splitting: comparison study between hand splitting and tablet cutter. Saudi Pharm. J. 2014, 22, 454-459. DOI: 10.1016/j. jsps.2013.12.014.

8. Vranić, E.; Uzunović, A. Influence of tablet splitting on content uniformity of lisinopril/ hydrochlorthiazide tablets. Bosn. J. Basic. Med. Sci. 2007, 7, 328-334. DOI: 10.17305/bjbms.2007.3022.

9. Dragomioiu, G. T. A. B; Ginghina, O.; Miron, D. S.; Bârca, M.; Popa D. E.; Hîrjau, M.; Lupuleasa, D.; Rădulescu, F. S. the influence of splitting on the in vitro release of metoprolol succinate from scored tablets. Farmacia. 2015, 63, 280-285.

10. Zhao, N.; Zidan, A.; Tawakkul, M.; Sayeed, V. A.; Khan, M. Tablet splitting: product quality assessment of metoprolol succinate extended release tablets. Int. J. Pharm. 2010, 401, 25-31. DOI: 10.1016/j.ijpharm.2010.09.004.

11. Ishitsuka, K.; Onuki, Y.; Takayama, K. [Change in the drug release behaviour of theophylline sustained-release tablets after division 
in two halves.] [in English] Yakugaku Zasshi. 2012, 132, 225-230. DOI: 10.1248/yakushi.132.225.

12. Margiocco, M. L.; Warren, J.; Borgarelli, M.; Kukanich, B. Analysis of weight uniformity, content uniformity and 30-day stability in halves and quarters of routinely prescribed cardiovascular medications. J. Vet. Cardiol. 2009, 11, 31-39. DOI: 10.1016/j. jvc.2009.04.003.

13. Volpe, D. A.; Gupta, A.; Ciavarella, A. B.; Faustino, P. J.; Sayeed, V. A.; Khan, M. A. Comparison of the stability of split and intact gabapentin tablets. Int. J. Pharm. 2008, 350, 65-69. DOI: 10.1016/j.ijpharm.2007.08.041.

14. European Pharmacopoeia 9th edition. EDQM Council of Europe: Strasburg, France. 2017.

15. Korsmeyer, R. W.; Gurny, R.; Doelker, E.; Buri, P.; Peppas, N. A. Mechanisms of solute release from porous hydrophilic polymers. Int. J. Pharm. 1983, 15, 25-35. DOI: 10.1016/03785173(83)90064-9.

16. Peppas, N. A. Analysis of Fickian and non-Fickian drug release from polymers. Pharm. Acta. Helv. 1985, 60, 110-111.

17. Moore, J. W.; Flanner, H. H. Mathematical comparison of dissolution profiles. Pharm. Technol. 1996, 20, 64-75.
18. Dissolution Testing of Immediate Release Solid Oral Dosage Forms; Guidance for Industry; U.S. Department of Health and Human Services, Food and Drug Administration, Center for Drug Evaluation and Research (CDER), U.S. Government Printing Office: Washington, DC, 1997.

19. Milanowski, B. Zastosowanie STATISTICA PROFILE UWALNIANIA w Pracach Badawczych i Przemyśle Farmaceutycznym. [in Polish] StatSoft Polska 2009, 39-52.

20. Costa, P.; Lobo, J. M. S. Modeling and comparison of dissolution profiles. Eur. J. Pharm. Sci. 2001, 13, 123-133. DOI: 10.1016/ S0928-0987(01)00095-1.

21. Green, G.; Berg, C.; Polli, J. E.; Barends, D. M.; Brown, W. E. Pharmacopeial standards for the subdivision characteristics of scored tablets. Pharma Times. 2010, 42, 15-24.

22. European Pharmacopeia 5th edition. EDQM Council of Europe: Strasburg, France. 2005.

23. Takka, S.; Sakr, A.; Goldberg, A. Development and validation of an in vitro-in vivo correlation for buspirone hydrochloride extended release tablets. J. Control. Release. 2003, 88, 147-157. DOI: 10.1016/S0168-3659(02)00490-X.

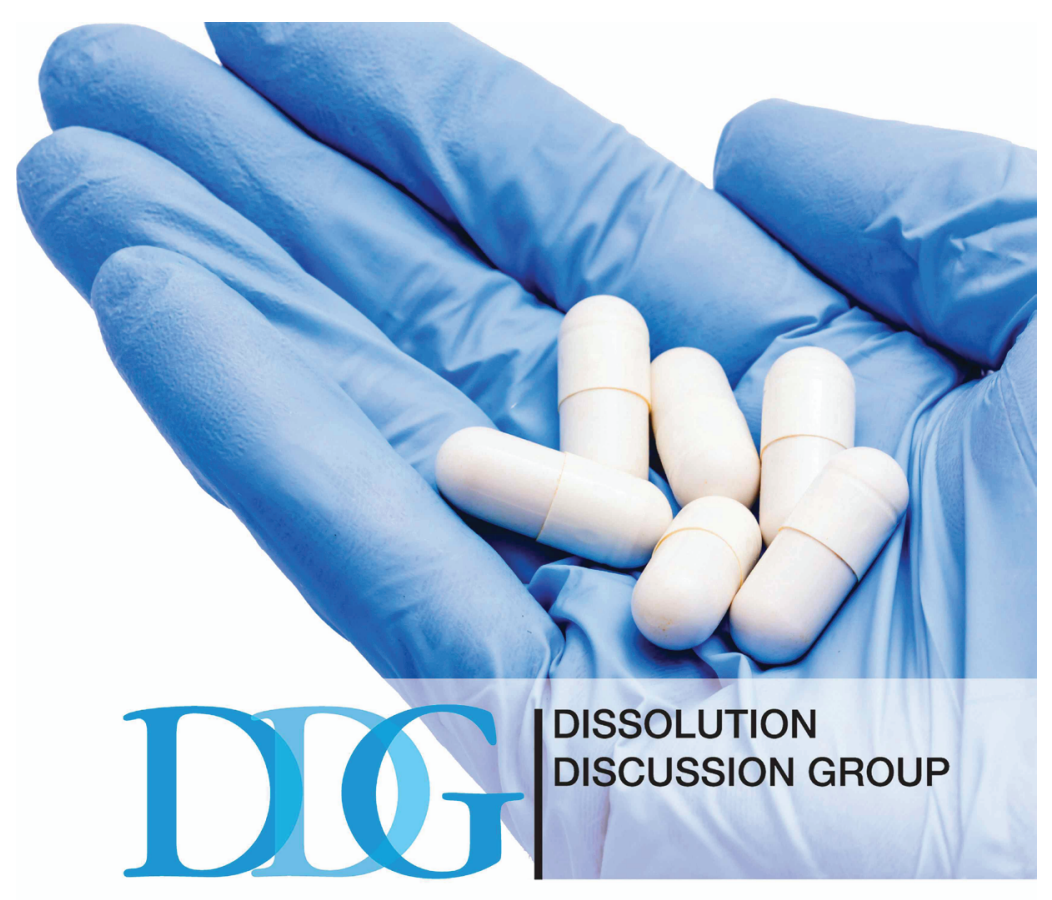

\section{For 20 years, the DDG has advanced the science of dissolution through the sharing of expertise}

\section{Thousands of questions and answers are at your fingertips in our online forum}

\section{Participate in quarterly online meetings or peruse our extensive archive of recorded meetings}

